\title{
Reliability of patient-reported outcome instruments in US adults with hemophilia: the Pain, Functional Impairment and Quality of life (P-FiQ) study
}

\author{
This article was published in the following Dove Press journal: \\ Patient Preference and Adherence \\ 19 September 2017 \\ Number of times this article has been viewed
}

\begin{abstract}
Christine L Kempton,'
Michael Wang, ${ }^{2}$ Michael

Recht, ${ }^{3}$ Anne Neff, ${ }^{4}$ Amy D

Shapiro, ${ }^{5}$ Amit Soni, ${ }^{6}$ Roshni

Kulkarni, ${ }^{7}$ Tyler W Buckner, ${ }^{2}$

Katharine Batt, ${ }^{8}$ Neeraj $\mathrm{N}$

lyer, ${ }^{9}$ David L Cooper'

'Departments of Pediatrics and Hematology and Medical Oncology, Emory University School of Medicine, Atlanta, GA, USA; ${ }^{2} \mathrm{Hemophilia}$ and Thrombosis Center, University of Colorado School of Medicine, Aurora, CO, USA; ${ }^{3}$ The Hemophilia Center. Oregon Health \& Science University, Portland, OR, USA; ${ }^{4} \mathrm{Hematology}$ and Medical Oncology, Cleveland Clinic, Cleveland, OH, USA; ${ }^{5}$ ndiana Hemophilia \& Thrombosis Center, Indianapolis, IN, USA; ${ }^{6}$ Center for Inherited Blood Disorders and $\mathrm{CHOC}$ Children's Hospital/UC Irvine, Orange, CA, USA; ${ }^{7} M S U$ Center for Bleeding and Clotting Disorders, Michigan State University, East Lansing, MI, USA ${ }^{8}$ Hematology and Oncology, Wake Forest School of Medicine, WinstonSalem, NC, USA; ${ }^{9}$ Clinical, Medical and Regulatory Affairs, Novo Nordisk Inc., Plainsboro, NJ, USA
\end{abstract}

Correspondence: Christine Kempton Emory University School of Medicine, 1760 Haygood Drive, Health Sciences Research Building Suite 340, Atlanta, GA 30322, USA

Tel +I 4047272846

Fax + I 404727 368I

Email ckempto@emory.edu
Background: Hemophilia is marked by frequent joint bleeding, resulting in pain and functional impairment.

Objective: This study aimed to assess the reliability of five patient-reported outcome (PRO) instruments in people with hemophilia (PWH) in a non-bleeding state.

Methods: Adult male PWH of any severity and inhibitor status, with a history of joint pain or bleeding, completed a pain history and five PRO instruments (EQ-5D-5L, Brief Pain Inventory v2 [BPI], International Physical Activity Questionnaire [IPAQ], Short Form 36 Health Survey v2 [SF-36v2], and Hemophilia Activities List [HAL]) during their routine comprehensive care visit. Patients were approached to complete the PRO instruments again at the end of their visit while in a similar non-bleeding state. Concordance of individual questionnaire items and correlation between domain scores were assessed using intra-class correlation coefficient (ICC).

Results: Participants completing the retest $(n=164)$ had a median age of 33.9 years. Median time for completion of the initial survey with PRO instruments was 36.0 minutes and for the five PRO instruments, median retest time was 21.0 minutes. The majority of participants had hemophilia A (74.4\%), were white and non-Hispanic (72.6\%), and self-reported arthritis/bone/ joint problems (61\%). Median/mean test-retest concordance was EQ-5D-5L 80.0\%/79.1\%, BPI 54.5\%/58.9\%, IPAQ 100\%/100\%, SF-36v2 77.8\%/76.4\%, and HAL 77.4\%/75.9\%. ICCs for test-retest reliability were EQ-5D-5L index 0.890; BPI - severity 0.950; BPI - interference 0.920; IPAQ total activity 0.940; SF-36v2 overall health 0.910 ; HAL total score 0.970.

Conclusion: All five PRO scales showed acceptable test-retest reliability in adult PWH. Therefore, the choice of instrument to be used for research or clinical care should be driven by instrument characteristics other than reliability.

Keywords: hemophilia, pain, patient-reported outcome, reliability

\section{Introduction}

Hemophilia is an inherited coagulopathy that results in acute bleeding, causing frequent pain and joint damage. ${ }^{1,2}$ Over time, recurring cycles of acute inflammation and swelling can lead to chronic pain and arthropathy. ${ }^{3,4}$ With an increase in life expectancy among people with hemophilia (PWH), there has been a greater focus on managing comorbidities associated with hemophilia, including pain. ${ }^{5}$ However, limited data are available on the prevalence and impact of pain in adult PWH.

Pain is inconsistently assessed both in clinical studies and in clinical practice. ${ }^{6,7}$ A survey of 22 European centers by the European Treatment Standardization Board 
found that although $67 \%$ of their patients experienced arthropathy and 35\% reported chronic pain, only eight of the centers used any formal pain assessment scales, and only two centers used the services of a pain specialist. ${ }^{8}$ Similarly, in a survey of 98 US hemophilia treatment centers (HTCs), only 15\% of responding centers reported having pain management as part of comprehensive care. ${ }^{9}$ Within hemophilia, some instruments may be better able to assess pain at early stages of joint disease (eg, soft tissue changes from acute or chronic synovitis, joint space distension from acute bleeding) when impact on functional impairment is less pronounced; others might be more appropriate at later stages of the disease to assess both pain and functional impairment associated with joint damage resulting in chronic arthropathy (eg, cartilage and bone changes, compromised range of motion, secondary muscle atrophy). While some studies in primary and secondary prophylaxis have employed quality-of-life (QoL) instruments, including disease-specific (eg, HAEMO-QoL and Hemophilia Activities List [HAL]) ${ }^{10-13}$ and generic (Short Form 36 Health Survey [SF-36]) scales, ${ }^{14-16}$ the baseline characteristics of these populations are unknown, and the studies are confounded by small sample size, which affects the generalizability of their findings. Additionally, although some generic and disease-specific patient-reported outcome (PRO) instruments have been used in studies of PWH, such as the HAL in the Netherlands, these have not been validated in adults with hemophilia in the USA. Currently, there are no data on Brief Pain Inventory Short Form (BPI) and EQ-5D-5L in US adult PWH and only limited data on SF-36v2 $2^{14-17}$ in this population. Previous studies of the EQ-5D among PWH, such as the cross-sectional assessment in the Hemophilia Experiences, Results and Opportunities (HERO) study ${ }^{18}$ and daily assessment in the Dosing Observational Study in Hemophilia (DOSE), ${ }^{19}$ used the 3-level scoring of the five domains, which may limit the instrument's ability to discriminate among PWH with milder phenotypes compared with the 5-level version. Because joint damage develops gradually over decades and is difficult to track, there is a clear need to identify and validate scales that can be used in clinical practice and research to assess pain and its relationship to functional impairment. ${ }^{6,7}$

The Pain, Functional Impairment and Quality of Life (P-FiQ) study was developed to assess the impact of pain on functional impairment and QoL in US adult PWH, with or without inhibitors, with joint bleeding, pain, or both. Previous surveys of adult PWH have been limited by opt-in bias and a lack of validated or comprehensive instruments that can capture both pain severity and interference with activities.
As a result, the impact of pain in adult PWH in the USA has not been captured. Participants in the P-FiQ study completed qualitative, generic surveys that assessed the prevalence and characteristics of acute and chronic pain and gathered information on the strategies employed to manage pain among PWH, as well as the impact of pain on function and QoL. These data were also used to validate the existing general and disease-specific QoL/pain scales used in the study. Test-retest reliability assessment is part of an extensive psychometric evaluation, including other measures of reliability testing (eg, item-total correlation) and measures of content validity (construct validity, known-group validity, etc), that will be presented in detail separately in parallel publications.

The overall aim of this article is to describe the P-FiQ study, with a particular focus on the survey methodology and demographic characteristics of study participants, and to provide an assessment of the reliability of generic and disease-specific PRO instruments. Evaluating the reliability of these PRO scales will provide critical information about whether these measures would be potentially suitable for use in research and clinical practice in the future in the US adult PWH population. Given the current lack of consensus on assessing pain and associated functional impairment, validating at least one scale may help provide support for using these PRO instruments in clinical practice and assessment of research study cohorts.

\section{Methods}

This was a non-interventional, cross-sectional study in which adult PWH who were attending their annual or other routine periodic comprehensive care visit were approached for study recruitment (NCT01988532). Given the potential for adult PWH complicated by joint bleeding or joint pain to experience bleeds in one of the six most common index joints (ankles, knees, elbows) at least once or twice a month with a period of treatment of several days or longer, ${ }^{20}$ the day-to-day variability in pain and health scores reported in adult PWH with inhibitors, and the potential for associated functional impairment that might persist related to the specific joint bleed past the end of treatment, it was determined that any assessment of test-retest reliability would need to be in a similar non-bleeding state. Furthermore, adult PWH often must travel up to 6-8 hours to reach an HTC, making it difficult to have multiple assessments over a period of days, particularly for working adults who need to take time off to travel to/from visits. For these reasons, the test-retest reliability assessment was planned to span the normal 3-4 hour routine outpatient comprehensive care visit at the HTC. 
The study protocol was approved by the local institutional review board (IRB) or central IRB. A list of each approving IRB is provided in Box S1. Written informed consent was obtained from participants by researchers before engaging in study-related activities. There were no follow-up visits, no treatment was specified or provided, and no productrelated information captured; therefore, no safety data were captured. Investigators were directed that any adverse drug reactions relating to treatment prior to the study visit should be reported using their standard procedures for reporting spontaneous adverse events.

\section{Recruitment and study population}

The overall P-FiQ study planned to capture 300 to 400 consecutive adult PWH in the USA. Of those enrolled, it was estimated that 250 to 300 adult PWH would complete the study and 120 to 150 would participate in the retest phase. To be eligible for inclusion, participants were required to be males aged 18 years and older with congenital hemophilia A or B with or without inhibitors and have a history of joint bleeding or joint pain. Individuals had to be visiting a treatment center for a routine comprehensive care visit while in a steady or non-bleeding state. Joint range of motion was assessed, and patients had to be capable of completing the survey in English. Exclusion criteria included previous participation in this study. Participants were recruited from October 2013 to October 2014 from 15 US HTCs, including some of the largest and smallest HTCs in the USA responding to feasibility questionnaires, with a mean (median) of 25.4 (28.0) participants per site. Each patient was assigned a unique study number to preserve patient confidentiality and compensated for participation in the study based on whether he completed the initial case report forms (CRFs), and additionally if he completed the five PRO instruments at the end of the visit.

\section{Test-retest process}

The study design is described in Figure 1. Prior to the start of the comprehensive care visit, adult PWH completed part 1 of the CRF, which included sociodemographic information (education, employment), current hemophilia treatment, acute and chronic pain characteristics/descriptors and treatments, and five PRO instruments: EQ-5D-5L with visual analog scale (VAS; recall period: "today"), BPI (recall period: the last 7 days), International Physical Activity Questionnaire Short Form (IPAQ; recall period: the last week), SF-36v2 (recall period: the last 4 weeks), and HAL (recall period: previous month). Details regarding selection of the PRO instruments, scoring, and development history have been previously reported with the primary results of the PRO analysis. ${ }^{21}$

During the comprehensive care visit, the physician or the physician's associate completed part 2 of the CRF for all participants, which included demographics, hemophilia characteristics (diagnosis, hemophilia history, treatment, comorbidities), functional status (based on the Centers for

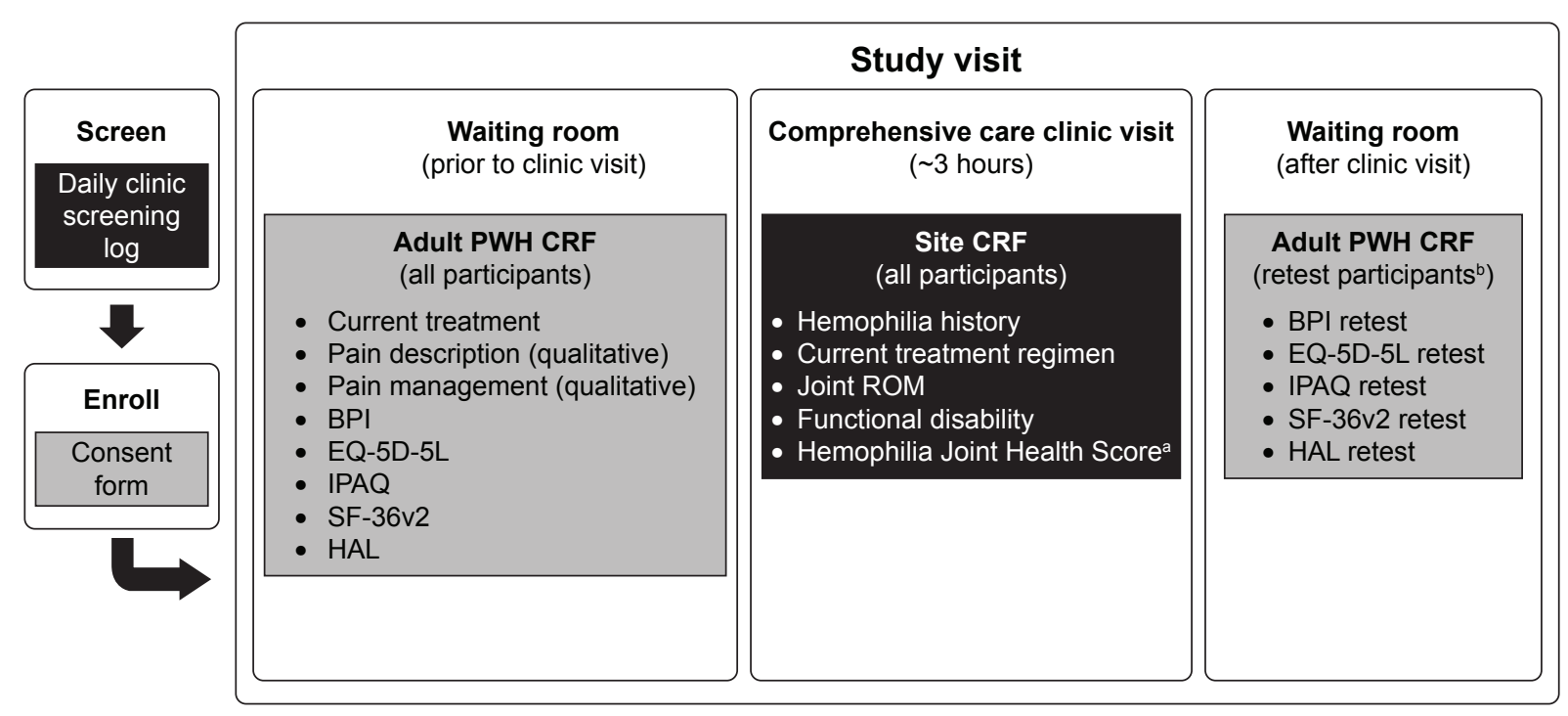

Figure I Pain, Functional Impairment and Quality of Life (P-FiQ) study design.

Notes: ${ }^{a} \mathrm{HJHS}$ can be captured for all subjects but will be optional based on physical therapist availability at the HTC site. ${ }^{b} \mathrm{Consecutively} \mathrm{enrolled} \mathrm{patients} \mathrm{will} \mathrm{be} \mathrm{offered}$ participation in the retest, until the retest cohort is complete.

Abbreviations: BPI, Brief Pain Inventory Short Form; CRF, case report form; HAL, Hemophilia Activities List; HJHS, Hemophilia Joint Health Score; HTC, hemophilia treatment center; IPAQ, International Physical Activity Questionnaire; PWH, people with hemophilia; ROM, range of motion; SF-36v2, Short Form 36 Health Survey v2. 
Disease Control and Prevention Universal Data Collection [CDC-UDC] template), joint range of motion (based on CDC-UDC template), and Hemophilia Joint Health Score v2.1, which was optional.

After completion of the comprehensive care visit, the first 150 participants who consented to complete the retest and were also in a similar non-bleeding state as when they took the initial PRO instruments completed part 3 of the CRF, which included the five PRO scales only: EQ-5D-5L with EQ-5D VAS, BPI, IPAQ Short Form, SF-36v2, and HAL. Time at initiation and completion of the test and retest were also captured for the participants.

For simplicity and to maximize the opportunity for sites with varying resources to try to recruit at least $75 \%$ of consecutive eligible participants, all CRFs were completed on paper. Data entry into the study database was performed by a contract research organization (Quintiles Outcome, Boston, MA, USA) with electronic edit checks and random data monitoring to assure accuracy of data entry.

\section{Statistical analyses}

Analyses were performed using SAS software, version 9.2 (SAS Institute Inc., Cary, NC, USA), and based upon a formal statistical analysis plan developed in advance of data collection. Data from participating sites were combined, and continuous variables were summarized by descriptive statistics as mean or median, standard deviation (SD) or quartiles (Q) (interquartile range [IQR], Q1 and Q3), and range (minimum and maximum). Total numbers of responses and percentages of responses for each category (including missing responses) and each item were calculated. Data from the study were either patient-reported or site-reported, which is clearly identified in tables/figures where applicable. The study was not powered for comparisons of reliability or validity assessments between the PRO instruments; since the different instruments included in this study contain different subsets of concepts, such analyses were not performed.

Test-retest reliability for the five scales has been primarily validated in other diseases (EQ-5D-5L, BPI, SF-36v2, and IPAQ) or in PWH other than US adults (HAL). ${ }^{22-32}$ Here, the test-retest reliability of the five PRO instrument scales was evaluated by correlating domain and/or global scores separately across the pre- and post-comprehensive exam visits (approximately 3-4 hours) using the intra-class correlation coefficient (ICC) assessing the source of variation as within target assuming one-way random effects (SAS proc glm, standard confidence intervals). ${ }^{33}$ The general minimum testretest reliability criterion for attributes that are expected to be relatively stable over time is 0.70 , however, an ICC greater than 0.80 is recommended by the International Prophylaxis Study Group. ${ }^{34,35}$ Concordance serves as a measure of agreement between different measuring or rating approaches, and was performed to assess agreement between initial pre-visit PRO responses and subsequent post-visit retest responses. Concordance of pre- and post-visit responses was calculated for percentage of overall agreement (SAS proc univariate, standard confidence intervals), based on individual item responses to each of the questionnaires, with higher values corresponding to a greater level of agreement.

\section{Results}

\section{Sociodemographics of retest population}

Altogether, out of the 544 individuals with hemophilia who were screened, 381 were enrolled in the overall P-FiQ study. The first 187 individuals were asked to participate in a retest at the completion of the comprehensive care visit in order to generate a retest population of at least 150 with complete responses. Of these 187 participants, 164 (87.7\%) completed the PRO retest, exceeding the target enrollment of 150; the additional 14 patients had completed the study with CRFs not yet received at the time the retest enrollment target was met and cohort closed, but were included in the retest analysis. The mean (median) age of the retest population was 37.1 (33.9) years (interquartile range [IQR] 26.9-46.0) (Table 1). The majority of the participants were white $(72.6 \%)$, married or in a long-term relationship (65.2\%), and did not live alone $(84.3 \%)$. Most had completed at least some college or graduate education (62.6\%), and were employed (80.8\%).

\section{Hemophilia history and comorbidities}

Most of the retest population had severe hemophilia $\mathrm{A}$ (74.4\%; Table 2), 6\% currently with inhibitors. The mean/ median age at inhibitor diagnosis was $15.9 / 12.0$ years (range, 0.3-53 years), and 15.9\% had either a current or past inhibitor titer higher than 0.5 Bethesda units $/ \mathrm{mL}$. When asked about comorbidities related to their condition, $61.0 \%$ of respondents reported arthritis/bone/joint problems. The majority of participants $(60.9 \%)$ were overweight $(32.9 \%)$ or obese $(28.0 \%)$, and the mean (SD) body mass index was $27.3(5.8) \mathrm{kg} / \mathrm{m}^{2}$ (range, 16.5-46.8). A history of intracerebral hemorrhage was reported by $13.4 \%$, occurring at a mean (SD) age of 14.5 (16.0) years; of these, 22.7\% reported that they continued to experience residual neurological problems. Among site-reported viral illnesses, hepatitis $\mathrm{C}$ virus (HCV) was more common than human immunodeficiency virus (HIV) (49.4\% vs $16.5 \%)$, and most individuals with 
Table I Sociodemographics of the retest population ( $n=164$, unless otherwise indicated)

\begin{tabular}{|c|c|}
\hline \multicolumn{2}{|l|}{ Age, years ${ }^{a}$} \\
\hline Mean & 37.1 \\
\hline Median & 33.9 \\
\hline (QI, Q3) & $(26.9,46.0)$ \\
\hline \multicolumn{2}{|l|}{ Race/ethnicity, ${ }^{\mathrm{a}} \mathrm{n}(\%)$} \\
\hline White, non-Hispanic & $119(72.6)$ \\
\hline Black & $20(12.2)$ \\
\hline White, Hispanic & $12(7.3)$ \\
\hline Other & I3 (7.9) \\
\hline \multicolumn{2}{|l|}{ Relationship status, ${ }^{\mathrm{b}} \mathrm{n}(\%)(\mathrm{n}=1 \mathrm{I})$} \\
\hline Married & $56(48.7)$ \\
\hline Long-term partner & $19(16.5)$ \\
\hline Single, never married & $28(24.3)$ \\
\hline Single, widowed & $2(1.7)$ \\
\hline Single, divorced & $10(8.7)$ \\
\hline \multicolumn{2}{|l|}{ Current living situation, ${ }^{\mathrm{b}} \mathrm{n}(\%)(\mathrm{n}=\mid 47)$} \\
\hline Lives with spouse/partner & $71(48.3)$ \\
\hline Lives with other family member & $39(26.5)$ \\
\hline Lives with roommates & $14(9.5)$ \\
\hline Lives alone & $23(15.6)$ \\
\hline \multicolumn{2}{|l|}{ Level of education, ${ }^{b} n(\%)(n=163)$} \\
\hline Did not complete high school & $18(11.0)$ \\
\hline High school graduate & $35(21.5)$ \\
\hline Trade/vocational school & $8(4.9)$ \\
\hline Some college/college graduate & $86(52.8)$ \\
\hline Postgraduate & $16(9.8)$ \\
\hline \multicolumn{2}{|l|}{ Employment status, ${ }^{\mathrm{b}, \mathrm{c}} \mathrm{n}(\%)(\mathrm{n}=135)$} \\
\hline \multicolumn{2}{|l|}{ Working } \\
\hline Full-time & $76(56.3)$ \\
\hline Part-time & $19(14.1)$ \\
\hline Self-employed & $14(10.4)$ \\
\hline \multicolumn{2}{|l|}{ Not working } \\
\hline Looking for employment & II (8.I) \\
\hline Retired & $7(5.2)$ \\
\hline Disabled short-term & $0(0.0)$ \\
\hline Disabled long-term & $9(6.7)$ \\
\hline Student & $20(14.8)$ \\
\hline Other & $3(2.2)$ \\
\hline
\end{tabular}

Notes: a'Site-reported; batient-reported; 'multiple selections allowed.

HCV or HIV were either currently receiving or had previously received antiretroviral therapy. Data on psychological conditions among the retest population were also captured: $17.7 \%$ had site-reported depression, and about $25 \%$ of these individuals were currently receiving treatment; $10.4 \%$ had site-reported anxiety, and of these individuals $29.4 \%$ were being treated; and $12.2 \%$ self-reported stress as a psychological condition. When asked about medications taken in the past 6-8 hours, $38.2 \%$ reported clotting factor or other hemophilia treatment for prophylaxis or bleeding, $38.8 \%$ had taken over-the-counter or prescription pain medications, $9.2 \%$ had taken a medication for depression or anxiety, and $42.8 \%$ reported that they had not taken any medications.
Table 2 Clinical characteristics of the retest population $(n=164$, unless otherwise indicated)

\begin{tabular}{|c|c|}
\hline \multicolumn{2}{|l|}{ Hemophilia type, ${ }^{\mathrm{a}} \mathrm{n}(\%)$} \\
\hline Hemophilia A & $122(74.4)$ \\
\hline Hemophilia B & $42(25.6)$ \\
\hline \multicolumn{2}{|l|}{ Severity, ${ }^{\mathrm{a}}$ (\%) } \\
\hline Mild with joint bleeding/pain (FVIII/FIX 5\%-I0\%) & $23(14.0)$ \\
\hline Moderate with joint bleeding/pain (FVIII/FIX I\%-<5\%) & $24(14.6)$ \\
\hline Severe $(\mathrm{FVIII/FIX}<1 \%)$ & $117(7 \mid .3)$ \\
\hline \multicolumn{2}{|l|}{ Current inhibitor titer, ${ }^{a} \mathrm{n}(\%)(\mathrm{n}=10)$} \\
\hline High (>5 BU/mL) & $9(90.0)$ \\
\hline Low $(<5 \mathrm{BU} / \mathrm{mL})$ & $I(I 0.0)$ \\
\hline \multicolumn{2}{|l|}{ Inhibitor history } \\
\hline Current or past inhibitor (>0.5 BU/mL), n (\%) & $26(15.9)$ \\
\hline Age at inhibitor diagnosis, mean/median, years & $15.9 / 12.0$ \\
\hline Peak inhibitor titer, mean/median, $\mathrm{BU} / \mathrm{mL}$ & $596.2 / 32.0$ \\
\hline \multicolumn{2}{|l|}{ Immune tolerance therapy, ${ }^{b} n(\%)(n=\mid 4)$} \\
\hline Not tolerized & $5(35.7)$ \\
\hline Tolerized & $8(57.1)$ \\
\hline Reoccurred & I (7.I) \\
\hline \multicolumn{2}{|l|}{ Body mass index category, ${ }^{\mathrm{a}} \mathrm{n}(\%)$} \\
\hline Normal/underweight $\left(<25 \mathrm{~kg} / \mathrm{m}^{2}\right)$ & $64(39.0)$ \\
\hline Overweight $\left(25-30 \mathrm{~kg} / \mathrm{m}^{2}\right)$ & $54(32.9)$ \\
\hline Obese $\left(\geq 30 \mathrm{~kg} / \mathrm{m}^{2}\right)$ & $46(28.0)$ \\
\hline \multicolumn{2}{|l|}{ Comorbidities, n (\%) } \\
\hline $\mathrm{HCV}^{\mathrm{a}}$ & $81(49.4)$ \\
\hline HCV treatment (past or present) & $29(35.8)$ \\
\hline $\mathrm{HIV}^{\mathrm{a}}$ & $27(16.5)$ \\
\hline HIV treatment (past or present) & $25(92.6)$ \\
\hline Arthritis/bone/joint problems ${ }^{\mathrm{b}}$ & $100(61.0)$ \\
\hline Diabetes $^{b}$ & $8(4.9)$ \\
\hline Cardiovascular disease $^{\mathrm{a}}$ & $28(17.1)$ \\
\hline Hypertension & $25(15.2)$ \\
\hline Coronary artery disease & $3(1.8)$ \\
\hline Other & $2(1.2)$ \\
\hline Other medical condition ${ }^{\mathrm{b}}$ & $36(22.0)$ \\
\hline \multicolumn{2}{|l|}{ Psychological conditions, n (\%) } \\
\hline Depression ${ }^{\mathrm{a}}$ & $28(17.7)$ \\
\hline Treatment with antidepressants & $7(25.0)$ \\
\hline Stress $^{\mathrm{b}}$ & $20(12.2)$ \\
\hline Anxiety & $17(10.4)$ \\
\hline Treatment with anxiolytics & $5(29.4)$ \\
\hline Other psychological condition ${ }^{\mathrm{b}}$ & II (6.7) \\
\hline \multicolumn{2}{|l|}{ History of $\mathrm{ICH},{ }^{\mathrm{a}} \mathrm{n}(\%)$} \\
\hline \multicolumn{2}{|l|}{$\mathrm{ICH}$} \\
\hline Yes & $22(13.4)$ \\
\hline No & $142(86.6)$ \\
\hline \multicolumn{2}{|l|}{$\mathrm{ICH}$ type } \\
\hline Epidural & $2(9.1)$ \\
\hline Subdural & $5(22.7)$ \\
\hline Intracerebral & $3(13.6)$ \\
\hline Subarachnoid & $0(0.0)$ \\
\hline Unknown & $12(54.5)$ \\
\hline \multicolumn{2}{|l|}{ Residual neurological problems } \\
\hline Yes & $5(22.7)$ \\
\hline No & $13(59.1)$ \\
\hline Unknown & $4(18.2)$ \\
\hline
\end{tabular}

Notes: aSite-reported outcome; ${ }^{\text {p} p a t i e n t-r e p o r t e d ~ o u t c o m e . ~}$

Abbreviations: BU, Bethesda unit; FVIII, factor VIII; FIX, factor IX; HCV, hepatitis $C$ virus; HIV, human immunodeficiency virus; $\mathrm{ICH}$, intracranial hemorrhage. 


\section{Concordance and reliability of PRO instruments}

The median time to complete the initial survey with PRO instruments (which included the pain survey) was 36.0 minutes, and the mean (median) time for the five PRO retests (without the pain survey) was 25.4 (21.0) minutes. The mean/median time between tests was 1.63 hours/1.52 hours. The mean/ median test-retest concordance percentage for each patient was calculated based on individual item responses to each of the questionnaires and ranged from $0 \%$ to $100 \%$ (Figure 2 ). The overall mean/median concordance of each PRO instrument was then calculated based upon individual concordance scores. Of the PRO instruments, BPI had the least concordance of test-retest item responses, with a mean (median) concordance of $58.9 \%(54.5 \%)$. The IPAQ had the highest mean/median concordance $(100 \% / 100 \%)$, followed by EQ-5D-5L index (79.1\%/80.0\%), SF-36v2 (76.4\%/77.8\%), and HAL (75.9\%/77.4\%).

The test-retest reliability of each questionnaire was assessed by the ICC (Table 3). All ICCs achieved the 0.70 minimum, except for the IPAQ subdomain "Walking activity", which had an ICC of 0.390. In general, the mental health subdomains of the SF-36v2 had lower ICCs relative to the physical health subdomains ("Mental health summary", 0.810; "Physical health summary", 0.940). When asked to rate the impact of pain on activities involving the upper and lower extremities on the HAL instrument, there was lower reliability among responses for the "Upper extremity activities" subdomain (0.870) compared with either the "Basic lower extremity activities" subdomain (0.950) or the "Complex lower extremity activities" subdomain (0.960).

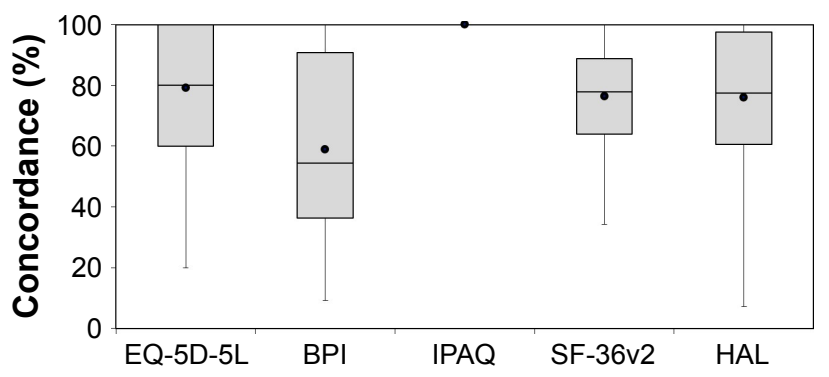

Figure 2 Concordance of individual item responses.

Notes: The test-retest concordance percentage for each patient was calculated based on individual item responses to each of the questionnaires and ranged from $7.1 \%$ to $100 \%$. Circles, mean; horizontal lines, median; shaded boxes, Ist and $3 \mathrm{rd}$ quartiles; vertical lines, minimum and maximum.

Abbreviations: BPI, Brief Pain Inventory; HAL, Hemophilia Activities List; IPAQ, International Physical Activity Questionnaire; SF-36v2, Short Form 36 Health Survey v2.
Table 3 Test-retest reliability of each PRO instrument

\begin{tabular}{|c|c|c|c|}
\hline & $\mathbf{n}$ & $I C C^{a}$ & $\begin{array}{l}95 \% \mathrm{Cl} \\
\text { (lower, upper) }\end{array}$ \\
\hline \multicolumn{4}{|l|}{ EQ-5D-5L } \\
\hline Index & 162 & 0.890 & $0.853,0.918$ \\
\hline \multicolumn{4}{|l|}{ BPI } \\
\hline Pain severity & 157 & 0.950 & $0.932,0.963$ \\
\hline Pain interference & 162 & 0.920 & $0.893,0.94 I$ \\
\hline \multicolumn{4}{|l|}{ IPAQ } \\
\hline Total physical activity & 142 & 0.940 & $0.918,0.956$ \\
\hline Vigorous activity & 105 & 0.950 & $0.927,0.966$ \\
\hline Moderate activity & 105 & 0.980 & $0.97 I, 0.986$ \\
\hline Walking activity & $|3|$ & 0.390 & $0.236,0.525$ \\
\hline \multicolumn{4}{|l|}{ SF-36v2 } \\
\hline Physical functioning & 162 & 0.940 & $0.919,0.956$ \\
\hline Role physical & 161 & 0.920 & $0.893,0.94 I$ \\
\hline Bodily pain & 162 & 0.890 & $0.853,0.918$ \\
\hline General health & 160 & 0.960 & $0.946,0.971$ \\
\hline Vitality & 162 & 0.860 & $0.814,0.895$ \\
\hline Social functioning & 163 & 0.830 & $0.776,0.872$ \\
\hline Role emotional & 162 & 0.760 & $0.687,0.818$ \\
\hline Mental health & 161 & 0.890 & $0.853,0.918$ \\
\hline Physical health summary & 163 & 0.940 & $0.919,0.956$ \\
\hline Mental health summary & 163 & 0.810 & $0.750,0.857$ \\
\hline Overall health & 163 & 0.910 & $0.880,0.933$ \\
\hline \multicolumn{4}{|l|}{$\mathrm{HAL}$} \\
\hline Lying/sitting/kneeling/standing & 161 & 0.950 & $0.932,0.963$ \\
\hline Functions of the legs & 161 & 0.960 & $0.946,0.970$ \\
\hline Functions of the arms & 161 & 0.900 & $0.866,0.926$ \\
\hline Use of transportation & 161 & 0.890 & $0.853,0.918$ \\
\hline Self-care & 161 & 0.780 & $0.712,0.834$ \\
\hline Household tasks & 161 & 0.910 & $0.879,0.933$ \\
\hline Leisure activities and sports & 160 & 0.930 & $0.906,0.948$ \\
\hline Upper extremity activities & 161 & 0.870 & $0.827,0.903$ \\
\hline Basic lower extremity activities & 161 & 0.950 & $0.932,0.963$ \\
\hline Complex lower extremity activities & 158 & 0.960 & $0.946,0.97 \mid$ \\
\hline Overall sum score & 162 & 0.970 & $0.959,0.978$ \\
\hline
\end{tabular}

Notes: The test-retest reliability of each questionnaire was assessed by ICC. aSite-reported outcome.

Abbreviations: BPI, Brief Pain Inventory Short Form; Cl, confidence interval; HAL, Hemophilia Activities List; ICC, intra-class correlation coefficient; IPAQ, International Physical Activity Questionnaire; PRO, patient-reported outcome; SF36v2, Short Form 36 Health Survey v2.

\section{Discussion}

Documenting patient-reported pain and the degree to which it affects quality of life for patients in the hemophilia community is a challenge for health care providers and HTCs. Scales such as the EQ-5D, SF-36, IPAQ, and HAL have been used previously in studies of PWH and validated in other diseases, ${ }^{24,26,29,31,36,37}$ but they have not been validated for use in adults with hemophilia in the USA. ${ }^{14-17}$ The P-FiQ study is the first study to use and validate the generic and disease-specific PRO instruments in US adults with hemophilia. Both the HERO study, ${ }^{18}$ the largest comprehensive global study to include pain and QoL assessments, and the 
DOSE study ${ }^{20}$ have applied the EQ-5D-3L and EQ-5D-VAS to the hemophilia patient population. Use of the EQ-5D-3L, however, was limited in only having three response levels to assess health status and might underestimate the impact of arthropathy by forcing patients to choose between the floor of none/no and the middle category of moderate/severe. ${ }^{38}$ The P-FiQ study therefore represents the first use of the new 5-level, validated version in hemophilia. ${ }^{28,39}$

To account for the presence of potential bleed-related confounding variables (bleed/no bleed at retest, time since last bleeding event, location and severity of last bleeding event, impact of bleed location on specific functional capabilities), the study was designed to allow only a short duration between the administration of the initial PRO test and the PRO retest (after the comprehensive care visit, expected $\sim 3-4$ hours). Test-retest reliability is typically performed by administering a second test at a later time, up to 4 weeks; however, based on an estimate from the DOSE study that $8 \%$ of days in a 3-month period in $\mathrm{PWH}$ with inhibitors are days associated with bleeding episodes, it is possible that responses would be different if the questionnaire retest was administered 1 or 2 weeks following an annual visit, even in patients without inhibitors. ${ }^{20}$ Because patients typically do not travel for comprehensive visits during or immediately following a bleed, there is potential that following the annual visit, the QoL of the patients would be different from that reported in the initial test, as it relates to the time frame of the different scales (EQ-5D, "today"; BPI, "the last week"; IPAQ, "the last 7 days"; ${ }^{1}$ SF-36v2 and HAL, "previous month" 25,33 ). Furthermore, potential long distances to an HTC could bias the population that would be available for a retest to those in more urban areas near the HTC. Unlike other chronic disorders in which day-to-day variability may be low, waiting 10 to 14 days for a retest in adult PWH might result in a falsely negative assessment of test reliability; therefore, participants in the retest phase were required to be in a similar non-bleeding state as in the initial test. It is conceivable that over the course of the comprehensive care visit, the health status of participants could change as medications that affect pain or physical function begin to wear off, or there could be an impact of the comprehensive visit itself (eg, testing of joint range of motion or gait) on functional abilities and QoL; however, this would likely be captured in the EQ-5D-5L, which assesses changes within the relevant time frame of "today". Based on the concordance and ICC data for this instrument, it is unlikely that there was a change in the bleeding state following the physical therapy portion of the comprehensive care visit. The BPI has a time frame of 1 week, and going through physical evaluation over several hours certainly could impact current pain, as well as indirectly impact worst/average pain experienced during the last week. BPI was associated with the lowest concordance, suggesting that even going through a routine visit could impact PRO instruments measuring pain-related concepts. Therefore, performing the retest $3-4$ hours after the conclusion of the comprehensive visit was judged to be ideal in balancing the potential for capturing a high percentage of consecutive patients with ensuring that patients present while in a similar enough general health state compared to the prior evaluation.

To represent patients with hemophilia who have joint bleeding and those affected by arthropathy, participants were included regardless of factor level. Additionally, the setting of a treatment center (HTC or elsewhere) for a comprehensive care visit was selected because it is generalizable to the adult PWH population. For purposes of assessing reliability and validity of the PRO instruments, it was simpler to conduct the study only with those who were able to complete the PRO instruments in English, because all of the instruments have been validated in hemophilia or another disease state in English. While mindful that a substantial proportion of PWH in the USA are Hispanic and that some of these patients might be more comfortable completing PRO instruments in Spanish, individuals with severe hemophilia A or B with reported Hispanic ethnicity account for only $\sim 12 \%$ to $14 \%$ of US PWH.

The primary limitation of the study design is the potential for respondent fatigue due to the numerous scales to be completed before the visit and then again during the retest phase. While it is possible that some participants may recall their survey responses due to the short time frame between the initial test and retest, there are over 100 questions across five PRO instruments, reducing the likelihood that response recall would impact concordance. There is also the possibility that some participants may try to rapidly complete the post-visit retest to be eligible for the compensation that was designed to alleviate the burden of completing the tests under such circumstances. However, based on the favorable concordance results, this does not appear to be a significant limitation. Similar high completion rates were also observed with the HERO study, an online patient survey of even greater length, ${ }^{18}$ which may be attributed to the direct relevance of the survey material to patients, the often prolonged periods spent waiting during comprehensive care visits, and the small amount of monetary compensation being offered to participants spending an additional hour or more. Furthermore, while 
retest reliability is one component of validity and a key endpoint of the study, additional analyses of internal consistency, item-total correlation, and construct validity from the P-FiQ study are also planned for the future. Another potential limitation is that the very act of asking patients for their measurements of quality of life pre- and post-test could positively impact the retest population; ${ }^{40}$ however, the ICC suggests that this is not relevant.

\section{Conclusion}

Test-retest analyses indicate that all five PRO instruments appear to be reliable in adult PWH. Each scale provides a different level of detail in describing the impact of hemophilia on pain and function in relation to both the number of questions and how specific they are; consequently, the scales have varied burdens of administration and utility. The retest reliability observations suggest that the choice of instrument to be used for research or clinical care should be driven by instrument characteristics other than reliability and that specific instruments should be tailored to the study design (eg, research on trends in overall study cohorts using short/ broad instruments like the EQ-5D-5L) or clinical need for specific outcome assessment (eg, pain management plan or physical therapy intervention based on detailed information provided by the BPI or HAL).

\section{Acknowledgments}

The clinical study analyzed was sponsored by Novo Nordisk A/S. Statistical analysis plan and analyses were provided by Jennifer James, MS at Quintiles Outcome under direction of Kari Kastango, PhD, and Pattra Mattox, MS. Writing assistance was provided by Shawn Keogan, PhD, of ETHOS Health Communications in Yardley, Pennsylvania, and was supported financially by Novo Nordisk Inc., Plainsboro, New Jersey, in compliance with international Good Publication Practice guidelines. The abstract of this paper was presented at the International Society of Thrombosis and Haemostasis 2015 congress as a poster presentation. The poster's abstract was published in the Journal of Thrombosis and Haemostasis.

\section{Disclosure}

CL Kempton: grant/research support from Novo Nordisk Inc.; consultant for Baxter, Biogen, CSL Behring, Kedrion. M Wang: consultant for Novo Nordisk Inc.. M Recht: grant/research support from Baxter, Biogen Idec, Novo Nordisk Inc., Pfizer; consultant for Biogen, Kedrion, Novo Nordisk Inc. A Neff: grant/research support from Novo Nordisk Inc.; consultant for
Alexion, Baxter, CSL Behring, Novo Nordisk Inc. AD Shapiro: consultant for Baxter BioScience, Novo Nordisk Inc., Biogen Idec, ProMetic Life Sciences, Kedrion Biopharma. A Soni: speakers bureau participant for CSL Behring and Novo Nordisk Inc.; consultant for Bayer. R Kulkarni: grant/research support from Novo Nordisk Inc., Biogen, Baxter, Bayer; consultant for Novo Nordisk Inc., Biogen, Bayer, Baxter, Kedrion, BPL, Pfizer. TW Buckner: consultant for Baxalta US, Genentech, and Novo Nordisk Inc. K Batt: grant/ research support from Novo Nordisk Inc.; scientific advisor for Precision Health Economics. NN Iyer and DL Cooper: employees of Novo Nordisk Inc., the sponsor of the study. The authors report no other conflicts of interest in this work.

\section{References}

1. Rodriguez-Merchan EC. Prevention of the musculoskeletal complications of hemophilia. Adv Prev Med. 2012;2012:201271.

2. Rodriguez-Merchan EC. Effects of hemophilia on articulations of children and adults. Clin Orthop Relat Res. 1996;(328):7-13.

3. Roosendaal G, Mauser-Bunschoten EP, De Kleijn P, et al. Synovium in haemophilic arthropathy. Haemophilia. 1998;4(4):502-505.

4. Roosendaal G, Lafeber FP. Pathogenesis of haemophilic arthropathy. Haemophilia. 2006;12 Suppl 3:117-121.

5. Mannucci PM, Schutgens RE, Santagostino E, Mauser-Bunschoten EP. How I treat age-related morbidities in elderly persons with hemophilia. Blood. 2009;114(26):5256-5263.

6. Humphries TJ, Kessler CM. The challenge of pain evaluation in haemophilia: can pain evaluation and quantification be improved by using pain instruments from other clinical situations? Haemophilia. 2013; 19(2):181-187

7. Riley RR, Witkop M, Hellman E, Akins S. Assessment and management of pain in haemophilia patients. Haemophilia. 2011;17(6): 839-845.

8. Holstein K, Klamroth R, Richards M, Carvalho M, Pérez-Garrido R, Gringeri A. European Haemophilia Therapy Standardization Board. Pain management in patients with haemophilia: a European survey. Haemophilia. 2012;18(5):743-752.

9. Shapiro A, Cooper DL. U.S. survey of surgical capabilities and experience with surgical procedures in patients with congenital haemophilia with inhibitors. Haemophilia. 2012;18(3):400-405.

10. Bullinger M, von Mackensen S, Fischer K, et al. Pilot testing of the 'Haemo-QoL' quality of life questionnaire for haemophiliac children in six European countries. Haemophilia. 2002;8 Suppl 2:47-54.

11. von Mackensen S, Bullinger M; Haemo-QoL Group. Development and testing of an instrument to assess the quality of life of children with haemophilia in Europe (Haemo-QoL). Haemophilia. 2004;10 Suppl 1: 17-25.

12. Pollak E, Muhlan H, von Mackensen S, Bullinger M; Haemo-Qol Group. The Haemo-QoL Index: developing a short measure for health-related quality of life assessment in children and adolescents with haemophilia. Haemophilia. 2006;12(4):384-392.

13. Baumgardner J, Elon L, Antun A, et al. Physical activity and functional abilities in adult males with haemophilia: a cross-sectional survey from a single US haemophilia treatment centre. Haemophilia. 2013;19(4):551-557.

14. Witkop M, Lambing A, Divine G, Kachalsky E, Rushlow D, Dinnen J. A national study of pain in the bleeding disorders community: a description of haemophilia pain. Haemophilia. 2012;18(3):e115-e119.

15. Witkop M, Lambing A, Kachalsky E, Divine G, Rushlow D, Dinnen J. Assessment of acute and persistent pain management in patients with haemophilia. Haemophilia. 2011;17(4):612-619. 
16. Khawaji M, Astermark J, Berntorp E. Lifelong prophylaxis in a large cohort of adult patients with severe haemophilia: a beneficial effect on orthopaedic outcome and quality of life. Eur J Haematol. 2012;88(4): 329-335.

17. den Uijl I, Biesma D, Grobbee D, Fischer K. Turning severe into moderate haemophilia by prophylaxis: are we reaching our goal? Blood Transfus. 2013;11(3):364-369.

18. Forsyth AL, Gregory M, Nugent D, et al. Haemophilia Experiences, Results and Opportunities (HERO) Study: survey methodology and population demographics. Haemophilia. 2014;20(1):44-51.

19. Neufeld EJ, Recht M, Sabio H, et al. Effect of acute bleeding on daily quality of life assessments in patients with congenital hemophilia with inhibitors and their families: observations from the dosing observational study in hemophilia. Value Health. 2012;15(6):916-925.

20. Young G, Solem CT, Hoffman K, et al. Capturing daily assessments and home treatment of congenital hemophilia with inhibitors: design, disposition, and implications of the Dosing Observational Study in Hemophilia (DOSE). J Blood Med. 2012;3:131-138.

21. Recht M, Neufeld EJ, Sharma VR, et al. Impact of acute bleeding on daily activities of patients with congenital hemophilia with inhibitors and their caregivers and families: observations from the Dosing Observational Study in Hemophilia (DOSE). Value Health. 2014;17(6): 744-748.

22. Kempton CL, Recht M, Neff A, et al. Impact of pain and functional impairment in US adult people with hemophilia (PWH): patient-reported outcomes and musculoskeletal evaluation in the pain, functional impairment, and quality of life (P-FiQ) study. Blood. 2015;126(23):39.

23. Shrout PE, Fleiss JL. Intraclass correlations: uses in assessing rater reliability. Psychol Bull. 1979;86(2):420-428.

24. Cleeland CS, Ryan KM. Pain assessment: global use of the Brief Pain Inventory. Ann Acad Med, Singapore. 1994;23(2):129-138.

25. van Genderen FR, Westers P, Heijnen L, et al. Measuring patients' perceptions on their functional abilities: validation of the Haemophilia Activities List. Haemophilia. 2006;12(1):36-46.

26. Cleeland CS. The Brief Pain Inventory: User Guide. MD Andersen Cancer Center. Houston, Texas. 2009. Available from: https://www. mdanderson.org/documents/Departments-and-Divisions/SymptomResearch/BPI_UserGuide.pdf. Accessed August 27, 2015.

27. de Andres Ares J, Cruces Prado LM, Canos Verdecho MA, et al. Validation of the Short Form of the Brief Pain Inventory (BPI-SF) in Spanish patients with non-cancer-related pain. Pain Pract. 2015; 15(7):643-653.

28. EuroQol Group. EQ-5D-5L User Guide: Basic Information on How to Use the EQ-5D-5L Instrument. Version 2.1. Netherlands: EuroQol Group; 2015. Available from: https://euroqol.org/wp-content/ uploads/2016/09/EQ-5D-5L_UserGuide_2015.pdf. Accessed August $27,2015$.
29. Naegeli AN, Tomaszewski EL, Al Sawah S. Psychometric validation of the Brief Pain Inventory-Short Form in patients with systemic lupus erythematosus in the United States. Lupus. 2015;24(13):1377-1383.

30. Ware J. User's Manual for the SF-36v2 Health Survey. Second edition. Montreal, QC, Canada: Quality Metric Inc.; 2007.

31. Craig CL, Marshall AL, Sjostrom M, et al. International physical activity questionnaire: 12-country reliability and validity. Med Sci Sports Exerc. 2003;35(8):1381-1395.

32. Groen W, van der Net J, Lacatusu AM, Serban M, Helders PJ, Fischer K. Functional limitations in Romanian children with haemophilia: further testing of psychometric properties of the Paediatric Haemophilia Activities List. Haemophilia. 2013;19(3):e116-e125.

33. van Genderen FR, van Meeteren NL, van der Bom JG, et al. Functional consequences of haemophilia in adults: the development of the Haemophilia Activities List. Haemophilia. 2004;10(5):565-571.

34. Doria AS, Babyn PS, Lundin B, et al. Reliability and construct validity of the compatible MRI scoring system for evaluation of haemophilic knees and ankles of haemophilic children. Expert MRI working group of the international prophylaxis study group. Haemophilia. 2006;12(5):503-513.

35. Altman DG. Practical Statistics for Medical Research. 1st ed: Chapman \& Hall/CRC; 1990

36. Kosinski M, Keller SD, Ware JE Jr, Hatoum HT, Kong SX. The SF-36 Health Survey as a generic outcome measure in clinical trials of patients with osteoarthritis and rheumatoid arthritis: relative validity of scales in relation to clinical measures of arthritis severity. Med Care. 1999;37(5 Suppl):MS23-MS39.

37. Keller S, Bann CM, Dodd SL, Schein J, Mendoza TR, Cleeland CS. Validity of the brief pain inventory for use in documenting the outcomes of patients with noncancer pain. Clin J Pain. 2004;20(5):309-318.

38. Harrison MJ, Davies LM, Bansback NJ, Ingram M, Anis $\mathrm{AH}$, Symmons DP. The validity and responsiveness of generic utility measures in rheumatoid arthritis: a review. J Rheumatol. 2008;35(4): 592-602.

39. Herdman M, Gudex C, Lloyd A, et al. Development and preliminary testing of the new five-level version of EQ-5D (EQ-5D-5L). Qual Life Res. 2011;20(10):1727-1736.

40. Velikova $\mathrm{G}$, Booth $\mathrm{L}, \mathrm{Smith} \mathrm{AB}$, et al. Measuring quality of life in routine oncology practice improves communication and patient well-being: a randomized controlled trial. J Clin Oncol. 2004;22(4):714-724. 


\section{Supplementary material}

Box SI List of independent ethics committees/institutional review boards

\begin{tabular}{|c|}
\hline I. Munson Medical Center \\
\hline I 105 Sixth Street \\
\hline Traverse City, MI 49684-2386 \\
\hline 2. Emory University \\
\hline 1599 Clifton Road, 5th Floor \\
\hline Atlanta, GA 30322 \\
\hline 3. St Vincent Hospital and Health Care Center, Inc. \\
\hline 8402 Harcourt Road, Suite 805 \\
\hline Indianapolis, IN 46260 \\
\hline 4. Western Institutional Review Board \\
\hline 3535 7th Avenue SW \\
\hline Olympia, WA 98502-5010 \\
\hline 5. Henry Ford Health System \\
\hline CFP-Basement 046 \\
\hline 2799 West Grand Boulevard \\
\hline Detroit, MI 48202-2689 \\
\hline (Chairperson: Dr Timothy Roehrs, PhD) \\
\hline 6. Vanderbilt University \\
\hline 504 Oxford House \\
\hline Nashville, TN 37232-43I5 \\
\hline 7. Western Institutional Review Board \\
\hline 1019 39th Avenue SE \\
\hline Puyallup, WA 98374 \\
\hline 8. University of Colorado Multiple \\
\hline Institutional Review Board \\
\hline |300| E |7th Place, Building 500, Room N32 I4 \\
\hline Aurora, CO 80045 \\
\hline
\end{tabular}

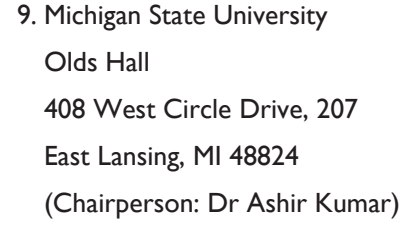

9. Michigan State University

Olds Hall

408 West Circle Drive, 207

East Lansing, MI 48824

(Chairperson: Dr Ashir Kumar)

10. Oregon Health \& Science University

318I SW Sam Jackson Park Road

Portland, OR 97239-3098

II. University of Minnesota

D528 Mayo Memorial Building

420 Delaware Street SE, MMC 820

Minneapolis, MN 55455

12. Rush University Medical Center

1653 West Congress Parkway

Chicago, IL 606I2-3833

13. Wake Forest University Health Services

Medical Center Boulevard

Winston-Salem, NC 27I57-1023

14. Georgetown University

37th and $O$ Streets, NW

Washington, DC 20057

\section{Publish your work in this journal}

Patient Preference and Adherence is an international, peer-reviewed, open access journal that focuses on the growing importance of patient preference and adherence throughout the therapeutic continuum. Patient satisfaction, acceptability, quality of life, compliance, persistence and their role in developing new therapeutic modalities and compounds to optimize clinical outcomes for existing disease states are major areas of interest for the journal. This journal has been accepted for indexing on PubMed Central. The manuscript management system is completely online and includes a very quick and fair peer-review system, which is all easy to use. Visit http://www. dovepress.com/testimonials.php to read real quotes from published authors. 